\title{
Hyperphenylalaninemia, $\mathrm{BH} 4$-deficient C
}

National Cancer Institute

\section{Source}

National Cancer Institute. Hyperphenylalaninemia, BH4-deficient C. NCI Thesaurus. Code C138173.

An autosomal recessive condition caused by mutation(s) in the QDPR gene, encoding dihydropteridine reductase. It is characterized by $\mathrm{BH}$ 4-defecient hyperphenylalanemia, depletion of dopamine and serotonin, and progressive cog nitive and motor deficits. 